\title{
Electron Probe Microanalysis of Actinide Elements: Our Past, Present, and Future
}

\author{
Karen E. Wright ${ }^{1}$
}

1. Idaho National Laboratory, Post Irradiation Examinations Dept., Idaho Falls, ID, USA.

Electron probe microanalysis (EPMA) of actinide elements plays a vital role in the characterization of nuclear fuel as it is primarily these elements that comprise the fuel. Although EPMA analysis of nuclear fuel has been extant since the early 1970's it was, and remains, extremely challenging for several reasons. Firstly, when actinides are present in irradiated nuclear fuel, they are accompanied by large $\beta$

and $\gamma$ radiation fields, which can cause several orders of magnitude increase in background noise as well as contribute to premature detector failure. Secondly, M-line actinide X-ray lines are used for analysis. These peaks suffer from abundant peak overlaps and a paucity of relevant physical parameters, particularly accurate mass absorption coefficients. Finally, analytical standards such as $\mathrm{Np}$ and $\mathrm{Pu}$ cannot be purchased. They must be made internally and do not have the robust analytical data and documentation that accompanies many geological standards (e.g. Smithsonian mineral standards).

The success of early attempts at actinide analysis was quite notable at the Institute for Transuranium Elements in Karlsruhe, Germany. In the early 1970's Giacchetti and Walker [1] had collaborated with the Cameca company to construct a custom-made instrument specifically for the analysis of irradiated nuclear fuels. This instrument combined the spectrometers of the CAMECA MS46 with the electron optical system of the CAMEBAX. It featured strategically-located $\mathrm{Pb}-\mathrm{W}$ shield plates to reduce specimen radiation on critical components, and a $220 \mathrm{~mm}$ Rowland circle with Johansson-geometry crystals to allow for greater peak separation. These workers found that the use of this shielding, as well as electronic discrimination could lower background radiation by a factor of $\sim 100$ (Fig. 1) [1,2]. Moreover, the use of very high emission currents (e.g. $250 \mathrm{nA}$ ) could increase the peak-to-background ratio by a factor of $\sim 10$, bringing the peak-to-background ratio of actinides in irradiated fuel comparable to that in unirradiated fuel [3]. Despite the very large Rowland circle and the use of Johansson-geometry crystals, peak overlaps problems were not eliminated completely. At that time, peak overlaps were handled manually. The count fraction contributed by the interfering peak was determined with standards and subsequently applied to all unknown acquisitions.

Actinide analysis today features similar hardware to Giacchetti and Walker [1], but with software and modeling techniques that were not available at that time. The Cameca SX100R is an EPMA based on the Cameca SX100 design, but provides shielding for 3 curies of gamma radiation at $0.75 \mathrm{MeV}$. This instrument is equipped with up to four wavelength-dispersive spectrometers and has no energy-dispersive spectroscopy detector. It has a $180 \mathrm{~mm}$ Rowland circle with Johann geometry crystals. One unusual addition to the crystal selection is the Quartz 1011. This is specifically used for actinide elements due to its increased resolution. For example, on the PET crystal, $\mathrm{U} \mathrm{M} \alpha$ at FWHM is $12 \mathrm{eV}$ compared to $9 \mathrm{eV}$ on the Quartz 1011 (Fig. 2). Despite resolution improvements imparted by the Quartz 1011 crystal, peak overlaps in the actinide spectrum are pernicious. $\mathrm{Pu} M \beta$ is selected due to the $\mathrm{Pu} M \alpha / \mathrm{U} M \beta$ overlap and $\mathrm{Am} \mathrm{M} \alpha$ is virtually coincident with $\mathrm{Np} \mathrm{M} \beta$ (Fig. 2). Cm M $\alpha$ overlaps with $\mathrm{Pu} \mathrm{M} \beta$ and $\mathrm{U}$ M $\gamma_{2}$ [4]. Fortunately software such as Probe for EPMA performs excellent overlap corrections complete with matrix corrections.

Analytical standards remain problematic. Thorium and uranium (as metal or oxide) can be purchased commercially, but for $Z \geq 93$, laboratories commonly synthesize their own standards. This can be difficult 
as these elements can occur in more than one oxidation state [5], thus precise temperature and atmosphere control during synthesis is essential. While oxide synthesis commonly results in porous materials, phosphate synthesis (e.g. $\mathrm{PuPO}_{4}$ ) has yielded promising results [6]. Recently attempts have been made to create virtual actinide standards in an effort to eliminate the problem of obtaining quality actinide standards $[7,8]$.

Because nuclear fuels are commonly $\mathrm{U}_{\mathrm{x}} \mathrm{O}_{\mathrm{y}}$ or $\mathrm{U}_{\mathrm{x}} \mathrm{O}_{\mathrm{y}} \mathrm{C}_{\mathrm{z}}$-based, future actinide research is focused around determining area peak factors and mass absorption coefficients of light elements in an actinide matrix.

\section{References:}

[1] M. Giacchetti and C.T. Walker, Electron Microscopy 3, (1980), p. 38.

[2] C Walker, J. Anal. Spec., 14 (1999), p. 447.

[3] M Perrot, PhD Thesis, Universite Paris Sud UFR Scientifique D’Orsay, (1995).

[4] H. Kleykamp, Z. Naturforsch 36a (1981), p. 1388.

[5] K Lieser, Nuclear and Radiochemistry, second edition, Wiley-VCH, Darmstadt, 462p.

[6] K Wright, et al., IOP Conference Series: Materials Science and Engineering, 304 (2018), p. 012020.

[7] C Merlet, et al., Microchim Acta 161, (2008), p. 427.

[8] A Moy, et al., IEEE Trans. Nucl. Sci. 61 (2014), p. 1977.

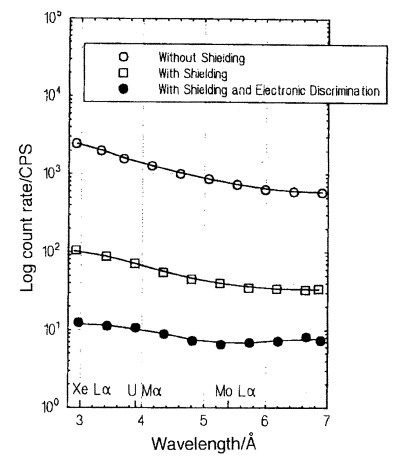

Figure 1. PET wavescan on sample with gamma field of $150 \mathrm{mR} / \mathrm{hr}$ at $30 \mathrm{~cm}$. Empty circles (no shielding), squares (with shielding), filled circles (with shielding and electronic discrimination). Excerpted from C. Walker [2].

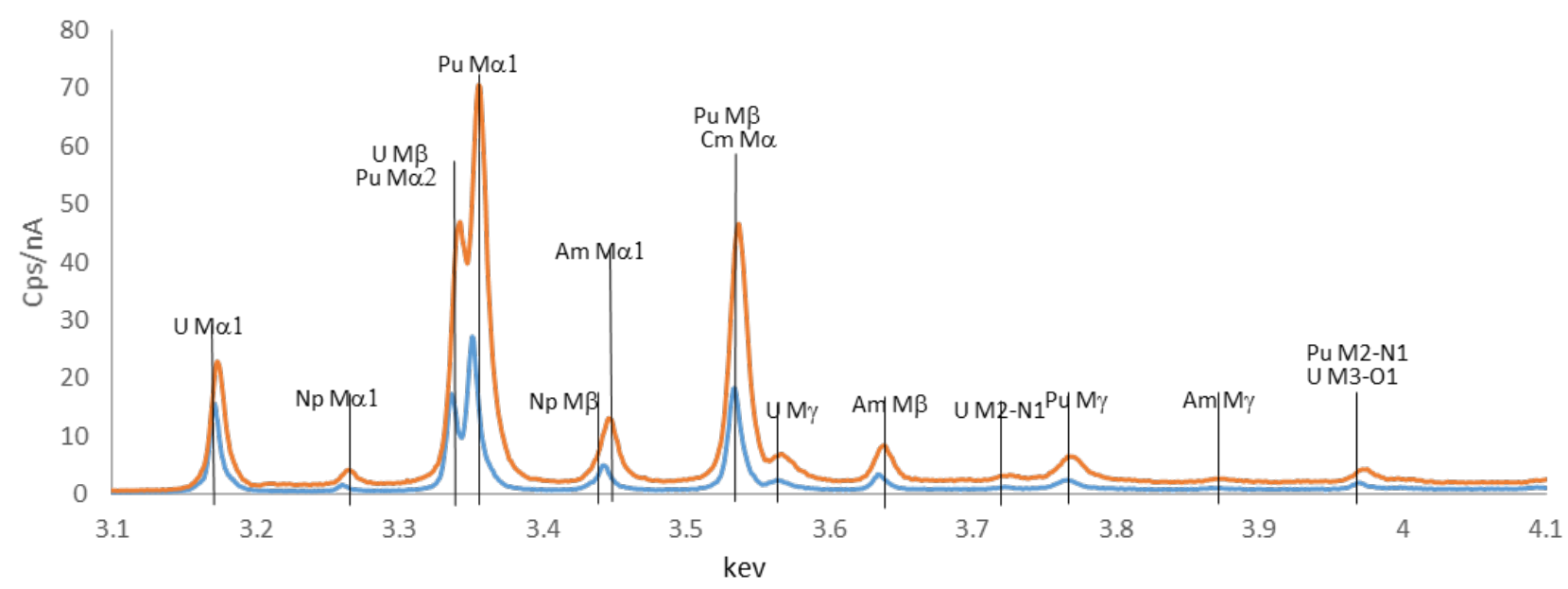

Figure 2. Wavescan with PET (orange) and Quartz 1011 (blue) on an irradiated metallic nuclear fuel whose pre-irradiation composition was $34.5 \mathrm{wt} \% \mathrm{U}, 28.1 \mathrm{wt} \% \mathrm{Pu}, 3.8 \mathrm{wt} \% \mathrm{Am}, 2.1 \mathrm{wt} \%$ $\mathrm{Np}, 31.5 \mathrm{wt} \% \mathrm{Zr}$. 